OPEN ACCESS

Edited by:

Peter Bader,

University Hospital Frankfurt, Germany

Reviewed by:

Luca Lo Nigro,

Azienda Ospedaliero Universitaria

Policlinico-San Marco, Italy

Jürgen Föll,

Children's University Hospital of

Regensburg, Germany

${ }^{*}$ Correspondence: Xiaojun Yuan

yuanxiaojun@xinhuamed.com.cn

Specialty section:

This article was submitted to

Pediatric Oncology,

a section of the journal

Frontiers in Oncology

Received: 22 July 2021 Accepted: 22 October 2021 Published: 15 November 2021

Citation:

Zhang D, Kaweme NM

Duan P, Dong Y and Yuan X

(2021) Upfront Treatment of Pediatric High-Risk Neuroblastoma With Chemotherapy, Surgery, and

Radiotherapy Combination: The CCCG-NB-2014 Protocol.

Front. Oncol. 11:745794. doi: 10.3389/fonc.2021.745794

\section{Upfront Treatment of Pediatric High-Risk Neuroblastoma With Chemotherapy, Surgery, and Radiotherapy Combination: The CCCG-NB-2014 Protocol}

\author{
Dongdong Zhang ${ }^{1,2}$, Natasha Mupeta Kaweme ${ }^{3}$, Peng Duan ${ }^{4}$, Youhong Dong ${ }^{2}$ \\ and Xiaojun Yuan ${ }^{1 *}$
}

${ }^{1}$ Department of Pediatric Hematology/Oncology, Xinhua Hospital Affiliated to Shanghai Jiao Tong University School of Medicine, Shanghai, China, ${ }^{2}$ Department of Oncology, Xiangyang No. 1 People's Hospital, Hubei University of Medicine, Xiangyang, China, ${ }^{3}$ Department of Hematology, Zhongnan Hospital Affiliated to Wuhan University, Wuhan, China, ${ }^{4}$ Department of Obstetrics and Gynaecology, Xiangyang No. 1 People's Hospital, Hubei University of Medicine, Xiangyang, China

Purpose: The Chinese Children's Cancer Group developed the CCCG-NB-2014 study to formulate optimal treatment strategies for high-risk (HR) neuroblastoma (NB). The safety and efficacy of this protocol were evaluated.

Method: Patients with newly diagnosed neuroblastoma and defined as HR according to the Children's Oncology Group study were included. They were treated with a combination of chemotherapy, surgery, and radiotherapy. The treatment-related toxicities, response rate, 3-year progression-free survival (PFS), and overall survival (OS) were analyzed.

Results: Of 159 patients enrolled between 2014 and 2018, 80 were eligible, including 19 girls and 61 boys, with a median age of 3.9 years (range 0.9-11). After a median followup of 24 months (range 3-40), the median OS was 31.8 months, and 3-year OS was 83.8\%. In multivariate analyses, the OS was affected by N-MYC amplification (hazard ratio 0.212 , 95\% confidence interval $(\mathrm{Cl}) 0.049-0.910 ; p=0.037$ ) and giant tumor mass (hazard ratio $0.197,95 \% \mathrm{Cl} 0.071-0.552 ; p=0.002$ ). The median 3-year PFS was 25.8 months, and 3-year PFS was $57.5 \%$. The univariate analysis showed that only the giant tumor mass was associated with the outcome. Of the 13 deaths, 11 died from the rapid progression of the disease and two from treatment-related toxicities. The most common adverse reaction was chemotherapy-induced hematological toxicity.

Conclusion: The PFS and OS reported in our study were similar to Western countries. The CCCG-NB-2014 protocol proved to be an efficient regimen with tolerable side-effect for the treatment of pediatric HR-NB.

Keywords: neuroblastoma, high risk, CCCG-NB-2014, overall survival, N-myc 


\section{INTRODUCTION}

Neuroblastoma (NB) is the most prevalent malignant extracranial solid tumor in children and may be considered to originate from the adrenal medulla or paravertebral sympathetic nervous system. NB generally occurs in children under the age of 15 , with a median diagnosis age of 17 months and an incidence of 10.2 per million $(1,2)$. The disease has a variable clinical presentation due to its marked tumor heterogeneity. NB could spontaneously regress with no symptoms; adversely, it can also progress rapidly with local invasion and metastasis. With the efforts of international multicenter cooperation groups, the 5 -year-related survival rate of NB was substantially improved between 1975 and 2009, from 54\% to $79 \%$ (3).

NB has a highly variable prognosis; therefore, developing a practical risk-classification algorithm to guide the treatment was necessary. Age less than 18 months was defined as a favorable prognosis as patients between 12 and 18 months of age achieved a longer survival (4). Tumor stage formulated by the International Neuroblastoma Staging System (INSS) could indirectly reflect tumor burden and the underlying tumor biology (5). Moreover, N-MYC amplification and tumor histopathological grade were biological prognostic factors $(6,7)$. On the basis of age at the initial diagnosis, the International Neuroblastoma Pathology Classification (INPC), INSS and N-MYC amplification status, and the Chinese Children's Cancer Group (CCCG) developed a riskstratification system and classified NB into three categories: low risk (LR), intermediate risk (IR), and high risk (HR).

The HR-NB had a relatively low initial response rate and poor prognosis than the LR-NB and IR-NB, with only $20 \%-35 \%$ survival rate (8-10). The main treatment method for HR-NB was intensive chemotherapy and surgical resection in the induction phase, external beam radiotherapy (XRT) and autologous bone marrow transplantation (ABMT) in the consolidation phase, and cis-retinoic acid (Cis-RA) in the maintenance phase. The ABMT could improve 5-year event-free survival (EFS) in patients with HR-NB (8); however, the treatment is a challenge for clinicians in many developing countries because of limited resources. Studies have shown that intensive chemotherapy could also improve clinical outcomes. Patients receiving chemotherapy without ABMT who achieved completed remission (CR) and very good partial remission (VGPR) had a better EFS than those who achieved partial remission or lower (8). However, $15 \%-20 \%$ of the patients with HR-NB were refractory to induction chemotherapy; approximately $50 \%$ of the patients with HR-NB either progress during the induction period or recurrence following $\mathrm{ABMT}(8,11,12)$. Therefore, developing a potent induction regimen was important for patients with HR-NB.

In 1994, the Memorial Sloan-Kettering Cancer Center (MSKCC) introduced a basic induction therapy, including high-dose cyclophosphamide combined with doxorubicin and vincristine. This protocol could help a majority of patients with HR-NB achieve CR or VGPR but with significant hematological and mucosal toxicities (13-15). From 1999 to 2010, the CCG-3891 study conducted by the Children's Oncology Group (COG) showed dose-intensive induction and consolidation could improve clinical outcomes, for example, chemotherapy regimen including high-dose cyclophosphamide or ifosfamide, cisplatin, doxorubicin, and etoposide $(8,12)$. The CCG-3891 induction chemotherapy incorporated four agents with nonresistance mechanisms of cytotoxicity absence of vincristine and increase the chemotherapy cycle to eight cycles, subsequently with the high incidence of grades 3 and 4 adverse effects. A phase II clinical trial (COG-ANBL02P1) in 2010 showed that topotecan combined with cyclophosphamide could decrease the rate of infectious complications, improve the response rate, and delay the progression time in patients with recurrent $\mathrm{NB}$ (16). Therefore, COG added two cycles of topotecan and cyclophosphamide combined with four cycles of N7 chemotherapy for the pilot induction of the newly diagnosed HR-NB in 2011 (COG-2011 pilot study); this protocol is confirmed to be safe and feasible for patients with HR-NB (17). The CCCG formulated the CCCG-NB-2014 protocol to explore the optimum induction therapy for $\mathrm{NB}$ on the basis of the MSKCC regimen, COG-ANBL02P1 study, and COG-2011 pilot study in 2014. We analyzed the response rate, toxicities, survival rate, and prognostic factors in patients with newly diagnosed HR-NB who were treated according to the CCCG-NB-2014 protocol in this prospective study.

\section{PATIENTS AND METHODS}

\section{Patient Eligibility and Study Design}

Patients below 18 years who were newly diagnosed with NB, and previously untreated, and with adequate performance and organ functions were enrolled between October 2014 and December 2018 in our study. The diagnosis of NB was based on tissue pathology or evidence of bone marrow involvement with increased urine vanillylmandelic acid (VMA) and serum nonspecific esterase (NSE) metabolites (5). Clinical staging was based on INNS and International Neuroblastoma Risk Group staging system $(5,18)$. The risk stratification was referred to COG $(11,19,20)$. Only patients defined as HR were eligible. The detailed inclusion criteria are listed in Table $\mathbf{1 .}$

Normally, patients underwent surgery and peripheral blood stem cell (PBSC) collection after four cycles of induction chemotherapy; subsequently, with four cycles of consolidation chemotherapy and radiotherapy, cis-retinoic acid (13-cis-RA) was used for maintenance therapy at the last stage. ABMT was recommended to be performed twice on the patients, before and after radiotherapy. In some cases, the operation was carried out ahead of schedule when clinicians needed the tumor tissue for a pathological investigation to confirm the diagnosis. In contrast, patients with giant tumor mass may receive more than four cycles of preoperative chemotherapy.

This study was approved by the Ethics and Scientific Committee of Xinhua Hospital Affiliated to Shanghai Jiao Tong University School of Medicine with approval number SHXH2021038 and was performed following Good Clinical Practice Guidelines and the Helsinki Declaration. All patients gave written informed consent before enrollment. All data were collected from the electronic medical record of Xinhua Hospital Affiliated to Shanghai Jiao Tong University School of Medicine. 
TABLE 1 | Definition of high-risk neuroblastoma according to the Children's Oncology Group study.

\begin{tabular}{|c|c|c|c|c|}
\hline Risk group & Age (year) & INPC grade & INSS grade & N-MYC \\
\hline \multirow{6}{*}{ High risk } & $>1$ & UFH & 2 & Positive \\
\hline & $>1$ & UFH & 3 & Negative \\
\hline & $>1$ & $\mathrm{FH}$ & 3 & Positive \\
\hline & $>1.5$ & UFH or $\mathrm{FH}$ & 4 & Positive or negative \\
\hline & $<1$ & UFH or FH & 3 or 4 & Positive \\
\hline & Any age & UFH or FH & $4 s$ & Positive \\
\hline
\end{tabular}

INPC, International Neuroblastoma Pathology Classification; INSS, International Neuroblastoma Staging System; FH, favorable histologic type; UFH, unfavorable histologic type.

\section{Treatment Response Evaluation}

Treatment response was evaluated by detecting NSE and VMA levels, changes in primary lesions and metastases, and assessment of the N-MYC copy number and the minimal residual disease (MRD). The scan obtained from magnetic resonance imaging or positron emission tomography was used for imaging examinations. Fluorescence in situ hybridization was for cytogenetic assessment. Bone marrow aspiration and immunotyping were performed to evaluate the MRD. Therapeutic evaluation was based on the International Neuroblastoma Response Criteria and divided into imaging remission and metabolic remission, including complete remission (CR), very good partial remission (VGPR), partial remission (PR), mixed response (MR), no response (NR), and progressive disease $(\mathrm{PD})(5,21)$.

\section{Toxicity}

Patients were examined by hematological and biochemical tests and electrocardiogram and echocardiogram before every cycle of chemotherapy. A hearing test must be performed before a cisplatin-based regimen. A diuretic renogram should be done to assess the renal function due to the use of high dose of cyclophosphamide. If two consecutive four-degree myelosuppressive events were observed after chemotherapy and the hemogram did not return to normal more than 1 week after supportive therapy, the drug dose must be modified in the next cycle of chemotherapy. The National Cancer Institute Common Terminology Criteria for Adverse Events version 4.0 was referred to as grade adverse reactions (22).

\section{Statistical Analysis}

A univariate analysis was performed for age, sex, N-MYC status, skeletal metastases, bone marrow invasion, and tumor mass $<10 \mathrm{~cm}$ to estimate the prognostic risk factors of the 3 year PFS by analysis of variance test. A multivariate COX regression analysis was performed to assess the effect of prognostic risk factor on OS. OS and PFS were estimated by the Kaplan-Meier curve. Statistical significance was determined by a $p$-value $<0.05$. IBM SPSS Statistic 24 software was used for statistical analysis.

\section{RESULTS}

\section{Patient Characteristic}

From October 2014 to December 2018, 159 patients newly diagnosed with NB were enrolled, including $31 \mathrm{LR}, 38 \mathrm{IR}$, and
90 HR. The patients with HR could not be contacted after discharge from the hospital. Thus, 80 patients were eligible for our study (Figure 1), whose clinical and biological characteristics are summarized in Table 2. As shown in the table, HR-NB occurs at the median age of 3.9, mostly after 1.5 years of age (90\%) and mainly in boys (68.86\%). Adrenal medulla or abdomen was still the common primary site $(82.5 \%)$. The common metastatic sites were bone marrow (44\%) and bone $(46 \%)$. Almost $97.5 \%$ of the patients had an elevated serum NSE level, but only $51.25 \%$ of the patients had urine VMA elevation and less than $57.5 \%$ of the patients had NMYC amplification.

\section{Treatment}

The detailed treatment schedules are summarized in Figure 2. All the patients received eight cycles of chemotherapy (Table 3). The efficacy was evaluated after every two cycles of chemotherapy. Carboplatin could be considered if children suffered from hearing impairment after treatment with a cisplatin-containing regimen; doxorubicin might be replaced by liposomal doxorubicin in the case of cardiac dysfunction. Generally, surgery and PBSC collection were performed after the fourth chemotherapy. ABMT was performed twice, one before and one after radiotherapy. Cis-RA was selected for the maintenance treatment for 6 months, with a routine dose of $160 \mathrm{mg} / \mathrm{m}^{2}$, administrated orally for 14 consecutive days each month. Trimethoprim-sulfamethoxazole was given for three consecutive days per week during treatment. Patients were requested to countercheck and followed up every 2-month after the end of treatment.

\section{Response and Outcome}

The response rate is shown in Table 4. After four cycles of chemotherapy, 19 patients (21.25\%) showed CR, 24 patients (30\%) showed VGPR, and 17 patients (21.25\%) showed PR. The overall objective remission rate (ORR; $\mathrm{CR}+\mathrm{VGPR}+\mathrm{PR})$ was $60 / 80(75 \%)$. Two patients died after four cycles of chemotherapy. After eight cycles of chemotherapy, the ORR was $61 / 78$ (78.25\%), of which the CR was $47.44 \%$ (37/78).

At a median follow-up of 24 months (range 3-40 months), 13 patients died due to disease progression. Two patients with skull and orbital metastasis experienced rapid progression and died in 3 months. One patient died of hemophagocytic syndrome. One patient died due to giant tumor rupture and massive blood loss in the short term after four cycles of chemotherapy. Three patients died of severe infection, one of whom died after two cycles of chemotherapy. Six patients died of widespread metastasis and 


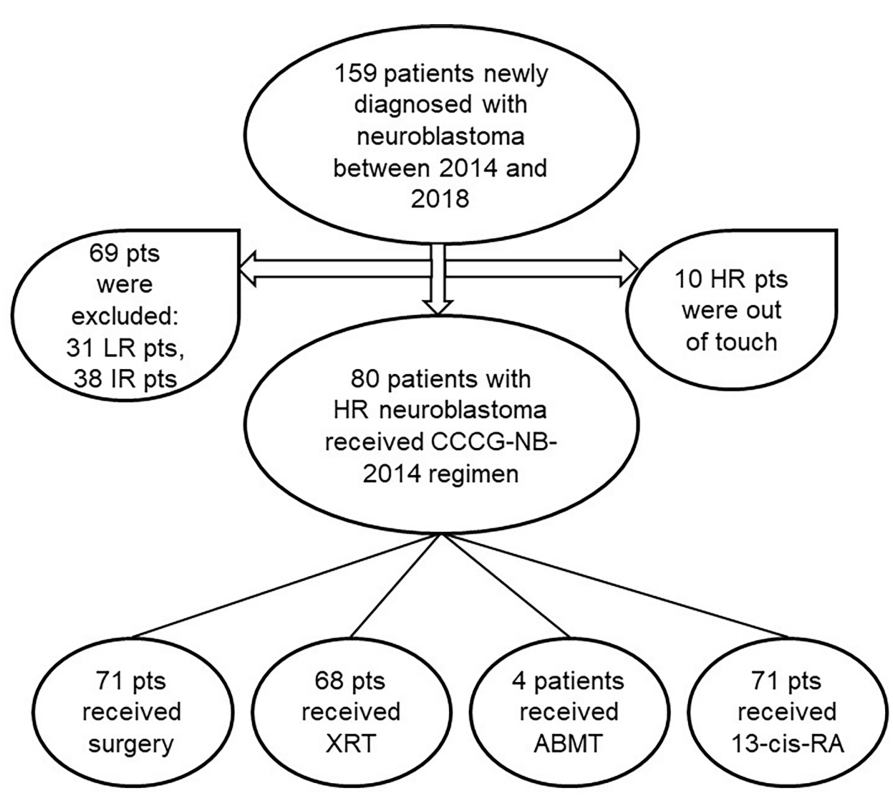

FIGURE 1 | Flow diagram of patients in the study. pts, patients; LR/IR/HR, low/intermediate/high-risk; XRT, external beam radiotherapy; ABMT, autologous bone marrow transplantation; Cis-RA, cis-retinoic acid.

TABLE 2 | Patient characteristics of study cohort.

\begin{tabular}{|c|c|}
\hline Characteristic & No. (\%) \\
\hline \multicolumn{2}{|l|}{ Age at diagnosis (years) } \\
\hline$<1.5$ & $8(10.00)$ \\
\hline$>1.5$ & $72(90.00)$ \\
\hline Median (range) & $3.9(0.9-11)$ \\
\hline \multicolumn{2}{|l|}{ Gender } \\
\hline Male & $61(68.86)$ \\
\hline Female & $19(31.14)$ \\
\hline \multicolumn{2}{|l|}{ Primary tumor site } \\
\hline Adrenal or abdominal & $66(82.50)$ \\
\hline Thoracic & $9(11.25)$ \\
\hline Neck and other sites & $5(6.25)$ \\
\hline \multicolumn{2}{|l|}{ Metastatic site at diagnosis } \\
\hline Bone marrow & $44(55.00)$ \\
\hline Bone & $46(57.50)$ \\
\hline Lymph node & $30(37.50)$ \\
\hline Other organs (liver, brain, lung) & $25(31.25)$ \\
\hline \multicolumn{2}{|l|}{ Serum NSE at diagnosis } \\
\hline Above normal & $78(97.50)$ \\
\hline Normal or unknown & $2(2.50)$ \\
\hline \multicolumn{2}{|l|}{ Urine VMA at diagnosis } \\
\hline Above normal & $41(51.25)$ \\
\hline Normal or unknown & $39(48.75)$ \\
\hline \multicolumn{2}{|l|}{ N-MYC } \\
\hline Positive & $16(20.00)$ \\
\hline Negative & $46(57.50)$ \\
\hline Unknown & $18(22.50)$ \\
\hline \multicolumn{2}{|l|}{ Tumor mass $>10 \mathrm{~cm}$} \\
\hline Positive & $8(11.25)$ \\
\hline Negative & $71(88.75)$ \\
\hline
\end{tabular}

Data are presented as median (range) for continuous variables and number (\%) for categorical variables. VMA, vanillylmandelic acid; NSE, nonspecific esterase; LOH, loss of heterozygosity. secondary multiple organ failure. The 3-year PFS was $57.5 \% \pm 4.2 \%$, and the median PFS was 25.8 months. The 3 -year OS was $83.8 \% \pm 3.1 \%$, and the median OS was 31.8 months (Figure 3).

\section{Adverse Events}

All patients were assessed, and grades 3 and 4 adverse events were recorded. The toxicity or tolerability is listed in Table $\mathbf{5}$ Hematological toxicity was the most common treatment-related toxicity. Twenty-four patients experienced febrile neutropenia, 13 of whom showed documented infection, including 11 bacterial infections and two fungal infections. About $20 \%$ of the patients required red blood cell transfusion and platelet transfusion. Four patients received the modified regimen (75\% dose) because of at least two four-degree myelosuppressive events. The incidence of hearing impairment was $22.5 \%$ $(18 / 80)$ in our study. The common side effect included hepatic and renal dysfunction, cardiac dysfunction, mucositis, vomit, diarrhea, and electrolyte disturbances. Two patients were infected with B19 parvovirus, resulting in severe anemia and acquired aplastic anemia. The rare treatment-related toxicity was secondary acute myeloid leukemia (sAML). One patient was diagnosed with sAML 3 years after the end of treatment; whereas, another was diagnosed 6 years later.

\section{Prognostic Factor}

A univariate analysis of the 3-year PFS showed that only tumor mass $>10 \mathrm{~cm}$ was the predictor $(p=0.025)$ (Table 6). A multivariate analysis of the 3-year OS, N-MYC amplification $(p=0.037)$, and tumor mass $>10 \mathrm{~cm}(p=0.002)$ achieved 


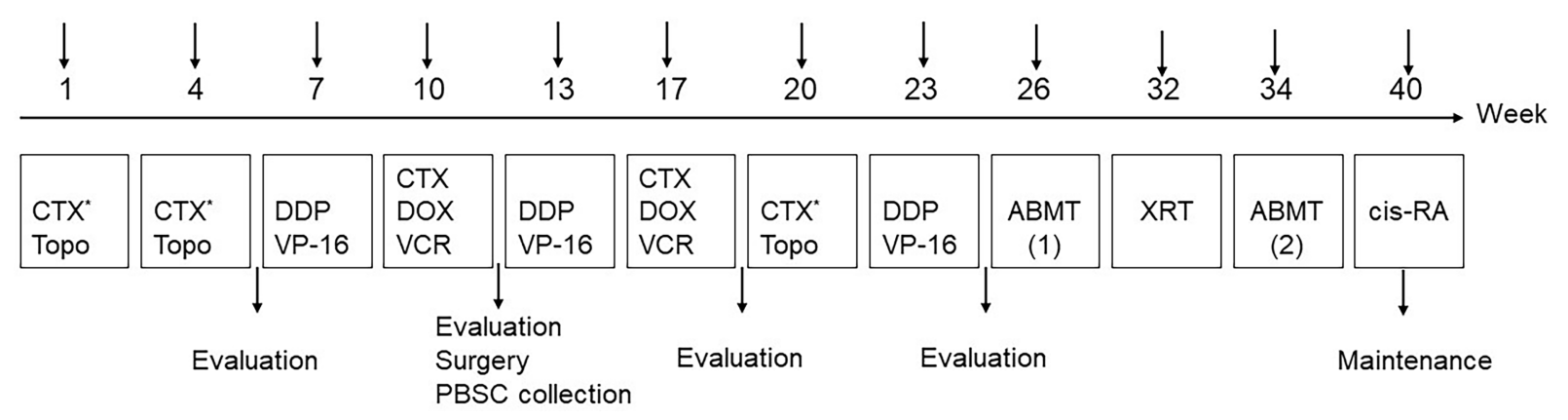

FIGURE 2 | Treatment schedule. PBSC, peripheral blood stem cells; XRT, external beam radiotherapy; 13-cis-RA: cis-retinoic acid.

TABLE 3 | CCCG-NB-2014 protocol.

\begin{tabular}{|c|c|c|c|}
\hline Agents & Dose & Route & Time \\
\hline \multicolumn{4}{|l|}{ CTX*+Topo } \\
\hline Cyclophosphamide* & 400 mg/m² (<12 kg: 13.3 mg/kg) & IV & D1-5 \\
\hline Topotecan & $1.2 \mathrm{mg} / \mathrm{m}^{2}$ & IV & D1-5 \\
\hline Irinotecan & $120 \mathrm{mg} / \mathrm{m}^{2}$ & & D1-3 \\
\hline \multicolumn{4}{|l|}{ DDP+VP-16 } \\
\hline Cisplatin & 50 mg/m² (<12 kg: 1.67 mg/kg) & IV & D1-4 \\
\hline Carboplatin & $560 \mathrm{mg} / \mathrm{m}^{2}(<12 \mathrm{~kg}: 18 \mathrm{mg} / \mathrm{kg})$ & & D1 \\
\hline Etoposide & $200 \mathrm{mg} / \mathrm{m}^{2}(<12 \mathrm{~kg}: 6.67 \mathrm{mg} / \mathrm{kg})$ & IV & D1-3 \\
\hline \multicolumn{4}{|l|}{ CTX+DOX+VCR } \\
\hline Cyclophosphamide & 1,200 mg/m² (<12 kg: 60 mg/kg) & IV & D1-2 \\
\hline Mesna & $420 \mathrm{mg} / \mathrm{m}^{2}(0,4$, and $8 \mathrm{~h}$ after CTX injection) & Push & D1-2 \\
\hline Doxorubicin & $25 \mathrm{mg} / \mathrm{m}^{2}(<12 \mathrm{~kg}: 0.83 \mathrm{mg} / \mathrm{kg})$ & IV & D1-3 \\
\hline Liposomal doxorubicin & $20 \mathrm{mg} / \mathrm{m}^{2}$ & & D1-3 \\
\hline \multirow[t]{3}{*}{ Vincristine } & 0.017 mg/kg (<12 months) & Push & D1-3 \\
\hline & $0.67 \mathrm{mg} / \mathrm{m}^{2}(<12$ months and $12 \mathrm{~kg})$ & & D1-3 \\
\hline & 0.022 mg/kg (>12 months, below 12 kg) & & D1-3 \\
\hline
\end{tabular}

statistical significance and was identified as predictive (Table 7). We did not analyze the relationship between ABMT and PFS/OS because only four patients received ABMT in our study.

\section{DISCUSSION}

NB accounted for only $7 \%$ of all pediatric malignant tumor, but the observed 5 -year mortality was $21 \%$ (3). NB had a high tumor heterogeneity; this unique characteristic was obvious in HR-NB, including variable clinical presentation and clinical outcome (23). Studies showed that early response in HR-NB was a predicator of better clinical outcome $(24,25)$. Furthermore, an increase in dose intensity of induction chemotherapy could improve the initial response rate (26). However, with the escalation in dose intensity, the incidence of adverse reactions also increased. For this reason, we initiated the CCCG-NB-2014 study to provide an optimal balance between efficacy and safety of the HRNB treatment.

In this pilot study, the induction regimen was formulated on the basis of the MSKCC regimen and the ANBL02P1 study.
Three cycles of topotecan plus cyclophosphamide (TOPO/CTX) combined with dose-intensive chemotherapy were introduced in this regimen. Two cycles of TOPO/CTX were performed at the beginning for induction and one cycle at the end for consolidation preparation. The pharmacokinetically guided topotecan dose was $1.2 \mathrm{mg} / \mathrm{m}^{2}$ according to the COG pilot study (17).

The most common adverse reaction was hematological toxicity, about $30 \%$ of the patients developed myelosuppression and were hospitalized, $13 \%$ patients were infected and needed positive anti-infection treatment, and about $20 \%$ patients needed transfusion. Febrile neutropenia and significant mucositis rarely occurred during the first two cycles of chemotherapy because when incorporated with topotecan, the cyclophosphamide dose was reduced, resulting in a shortened hospital stay. TOPO/CTX treatment-related toxicities were tolerable and manageable in our study, which was similar to that of the previous study (16) and further indicated that the first two cycles of topotecan plus cyclophosphamide were feasible for the initial induction. Hearing impairment was another adverse reaction that required special attention, with $22.5 \%$ of the patients developing this symptom after cisplatin-containing chemotherapy. In general, we considered carboplatin, which 
TABLE 4 | Induction treatment response after four- and eight-cycle chemotherapy.

\begin{tabular}{lcc}
\hline Treatment response & After $\mathbf{4}$ cycles [No. (\%)] & After 8 cycles [No. (\%)] \\
\hline CR & $19(23.75)$ & $37(47.44)$ \\
VGPR & $24(30.00)$ & $16(20.51)$ \\
PR & $17(21.25)$ & $8(10.25)$ \\
MR & $7(8.75)$ & - \\
NR & $5(6.25)$ & - \\
PD & $6(7.50)$ & $12(15.38)$ \\
Death & $2(2.50)$ & $5(6.41)$
\end{tabular}

Primary tumor resection was adopted before chemotherapy in patients evaluated as $C R$ in the "four-cycle" group. CR, complete remission; VGPR, very good partial remission; PR, partial remission; $M R$, mixed response; NR, no response; $P D$, progressive disease.

had less ototoxicity, in such a case. Extension of duration of cisplatin infusion was confirmed to be invalid (27). The intravenous administration of sodium thiosulfate $6 \mathrm{~h}$ after the discontinuation of cisplatin could be helpful in decreasing ototoxicity (28). Two patients, who experienced B19 virus infection possibly due to hypoimmunity after chemotherapy, presented with refractory anemia, and subsequent aplastic anemia. This is an indication that we also need to pay attention to virus infections during the induction phase. Secondary malignancy was a rare long-time side effect. The diagnosis of sAML 3 years after the end of treatment in two patients could be related to the mutagenic potential of alkylating agents (29), which may provide a rationale for reducing the number of cycles of dose-intensive chemotherapy and the necessity of ABMT. Only four patients underwent dose modification (reduction to 75\%) after the development of two consecutive four-degree myelosuppressive events. Considering the limited resource and expensive fee, only four patients underwent ABMT in our study. On the whole, the regimen was manageable and safe.

Within the context of the induction regimen, the patients achieved an overall response rate of $78.25 \%$ after eight cycles of induction chemotherapy, with $68 \%$ patients showing CR or VGPR. The ORR in our study was similar to the response rate (84\%) in the ANBL02P1 induction regimen, but we achieved a
TABLE 5 | Treatment-related toxicities after induction therapy.

\begin{tabular}{lc}
\hline Toxicity & No. (\%) \\
\hline Febrile neutropenia & $24(30.00)$ \\
Documented infection & $13(16.25)$ \\
Red blood cell transfusion & $16(20.00)$ \\
Platelet transfusion & $15(18.75)$ \\
Hearing impairment & $18(22.50)$ \\
Elevated liver enzymes & $12(15.00)$ \\
Renal dysfunction & $7(8.75)$ \\
Cardiac dysfunction & $5(6.25)$ \\
Mucositis & $3(3.75)$ \\
Vomit, diarrhea, electrolyte disturbances & $6(7.50)$ \\
Coagulation disorders & $9(11.25)$ \\
Dose modifications & $4(5.00)$ \\
B19 virus infection & $2(2.50)$ \\
Secondary acute myeloid leukemia & $2(2.50)$
\end{tabular}

better CR and VGPR (68\% vs. 48\%) (16). In the COG pilot study, PBSC collection was started after two cycles of CTX/ TOPO. The European Bone Marrow Transplant registry demonstrated that the CR of metastases before ABMT was a predictor of a better clinical outcome (30). Our result showed that $53.75 \%$ of the patients achieved CR or VGPR after four cycles of chemotherapy (Table 4); therefore, we harvested the PBSC after two cycles of CTX/TOPO and two cycles of doseintensive chemotherapy. More importantly, the PBSCs were less likely contaminated by tumor cells. The multivariate analysis indicated that the 3-year OS correlated with the $\mathrm{N}$ MYC amplification and tumor mass $>10 \mathrm{~cm}$ and was unrelated to age, stage, and metastasis site. However, two patients with skull and orbital metastasis died in a short time. Our result and other studies suggested skull and orbital metastases may be associated with poor prognosis (31). However, a large number of patients are still needed for further verification.

The pretreatment risk stratification of patients was essential and important for clinicians. The limitation of our study was that the diploid DNA index and the chromosome variation including $1 \mathrm{p}$ or $11 \mathrm{q}$ loss of the heterozygosity status were not detected to
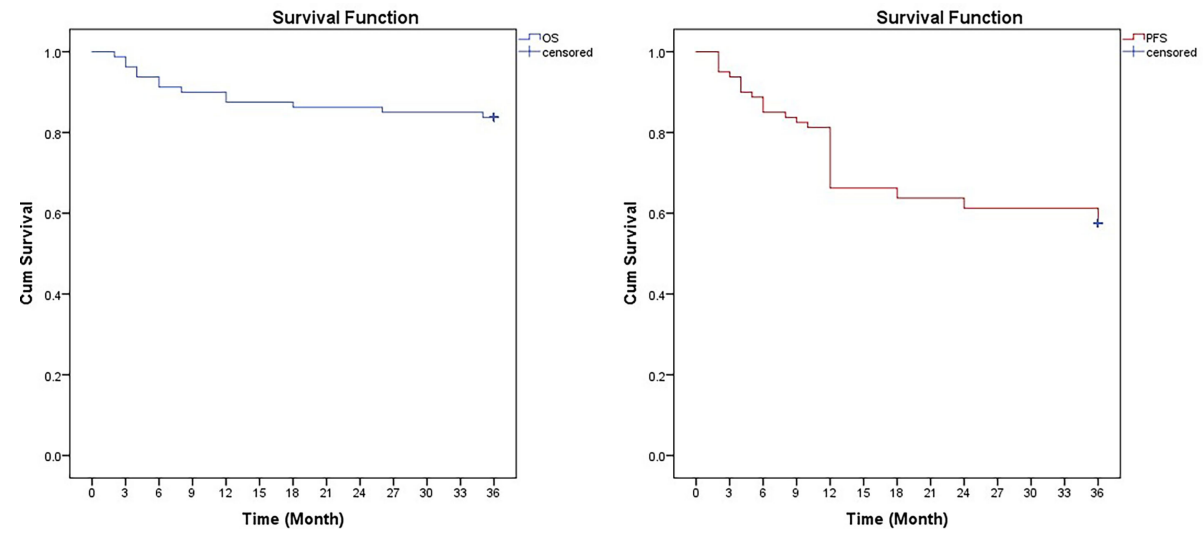

FIGURE 3 | Kaplan-Meier curves for overall survival (OS) and progression-free survival (FPS) in 80 children with high-risk neuroblastoma treated with CCCG-NB-2014. 
TABLE 6 | Univariate analysis of prognostic factors for 3-year PFS in 80 patients.

\begin{tabular}{lcc}
\hline Characteristic & Univariate analysis & 95\% Cl of 3-year EFS \\
\hline Age & & \\
$\quad>1$ & $23.80 \pm 12.62$ & $20.79-26.81$ \\
$<1$ & $20.70 \pm 14.52$ & $9.61-30.39$ \\
Sex & $22.87 \pm 12.97$ & $19.55-26.19$ \\
$\quad$ Male & $24.79 \pm 12.60$ & $18.71-30.87$ \\
$\quad$ Female & $20.91 \pm 12.81$ & $15.23-26.59$ \\
N-MYC amplification & $24.24 \pm 12.83$ & $20.87-27.62$ \\
$\quad$ Positive & $22.96 \pm 12.55$ & \\
$\quad$ Negative & $23.97 \pm 13.52$ & $19.43-26.49$ \\
Skeletal metastases & & $18.82-29.11$ \\
$\quad$ Positive & $21.88 \pm 12.99$ & $17.78-25.98$ \\
$\quad$ Negative & $24.85 \pm 12.65$ & $20.74-28.95$ \\
Bone marrow invasion & & \\
$\quad$ Positive & $14.33 \pm 13.82$ & $3.7-24.96$ \\
$\quad$ Negative & $24.46 \pm 12.34$ & $21.54-27.39$ \\
Tumor mass $>10 \mathrm{~cm}$ & & 0.372 \\
$\quad$ Positive & & 0.739 \\
$\quad$ Negative & & 0.304 \\
\hline
\end{tabular}

TABLE 7 | Multivariate analyses of prognostic factors for overall survival.

\begin{tabular}{lcc}
\hline & Hazard ratio $\mathbf{( 9 5 \% ~ C l )}$ & $\boldsymbol{p}$-value \\
\hline Age $>1$ & $2.540(0.826-7.812)$ & 0.104 \\
N-MYC amplification & $0.212(0.049-0.910)$ & 0.037 \\
Tumor mass $>10 \mathrm{~cm}$ & $0.197(0.071-0.552)$ & 0.002 \\
Bone marrow invasion & $1.807(0.888-3.678)$ & 0.103 \\
Skeletal metastases & $0.495(0.203-1.203)$ & 0.121 \\
\hline
\end{tabular}

the limited resources. This caused our 2014 HR stratification criteria to differ from the latest COG stratification and INPC criteria $(32,33)$. Previous studies showed that myeloablative therapy and ABMT were related to EFS (8); we did not perform correlation analysis due to the limited number of patients.

The novel therapies provided more choices for the treatment of HR-NB. ${ }^{131}$ I-labeled metaiodobenzylguanidine ( $\left.{ }^{131} \mathrm{I}-\mathrm{MIBG}\right)$ combined with topotecan was found to be effective in improving EFS and OS in patients with primary refractory HR-NB (34). The ${ }^{131}$ I-MIBG might develop as the first-line consolidation therapy in the future (35). Anti-GD-2 immunotherapy combined with interleukin 2 and Cis-RA was confirmed to be successful in eradicating the MRD in maintenance phase (36) and could significantly improve the clinical outcome (37). AntiGD-2 monoclonal antibody was recommended for the maintenance therapy by CCCG although it was unlisted in China. ALK somatic mutation or gene amplification was observed in $15 \%$ of the patients with NB and $30 \%$ with NBderived cell lines. Blocking the ALK mutation resulted in the inhibition of NB cell viability. This may provide new clinical insights into patients with $\mathrm{NB}$ with $A L K$ mutation or gene amplification $(38,39)$. Some other emerging therapies like Aurora kinase A inhibitor (40), programmed death-1 inhibitor, and chimeric antigen receptor $\mathrm{T}$ cell were also promising therapies under study $(41,42)$.

In conclusion, the CCCG-2014-NB protocol was efficient in improving the clinical outcome. The treatment-related toxicities were tolerable and manageable, and this regimen has the potential to be recommended as the upfront treatment for the newly diagnosed HR-NB.

\section{DATA AVAILABILITY STATEMENT}

The raw data supporting the conclusions of this article will be made available by the authors, without undue reservation.

\section{ETHICS STATEMENT}

This study was approved by the Ethics and Scientific Committee of Xinhua Hospital Affiliated to Shanghai Jiaotong University School of Medicine and was performed according to the Good Clinical Practice Guidelines and the Helsinki Declaration. Written informed consent to participate in this study was provided by the participants' legal guardian/next of kin.

\section{AUTHOR CONTRIBUTIONS}

$\mathrm{XY}$ provided the direction of the study. DZ collected and analyzed the data and wrote the manuscript. YD helped collect the data. NK and PD revised the manuscript. All authors contributed to the article and approved the submitted version. 


\section{FUNDING}

This work was supported by the Innovative Research Program of Xiangyang No. 1 People’s Hospital (Grant number: XYY2021Q02).

\section{REFERENCES}

1. London WB, Castleberry RP, Matthay KK, Look AT, Seeger RC, Shimada H, et al. Evidence for an Age Cutoff Greater Than 365 Days for Neuroblastoma Risk Group Stratification in the Children's Oncology Group. J Clin Oncol (2005) 23(27):6459-65. doi: 10.1200/JCO.2005.05.571

2. Ries L, Smith MA, Gurney JG. Cancer Incidence and Survival Among Children and Adolescents. United States SEER Prog 1975-1995 (1999) 1-15. doi: $10.1515 / 9783110895797.1$

3. Ward E, DeSantis C, Robbins A, Kohler B, Jemal A. Childhood and Adolescent Cancer Statistics, 2014. CA: Cancer J Clin (2014) 64(2):83-103. doi: $10.3322 /$ caac. 21219

4. Schmidt ML, Lal A, Seeger RC, Maris JM, Shimada H, O'Leary M, et al. Favorable Prognosis for Patients 12 to 18 Months of Age With Stage 4 Nonamplified MYCN Neuroblastoma: A Children's Cancer Group Study. J Clin Oncol (2005) 23(27):6474-80. doi: 10.1200/JCO.2005.05.183

5. Brodeur GM, Pritchard J, Berthold F, Carlsen NL, Castel V, Castelberry RP, et al. Revisions of the International Criteria for Neuroblastoma Diagnosis, Staging, and Response to Treatment. J Clin Oncol (1993) 11(8):1466-77. doi: 10.1200/JCO.1993.11.8.1466

6. Seeger RC, Brodeur GM, Sather H, Dalton A, Siegel SE, Wong KY, et al. Association of Multiple Copies of the N-Myc Oncogene With Rapid Progression of Neuroblastomas. N Engl J Med (1985) 313(18):1111-6. doi: 10.1056/NEJM198510313131802

7. Shimada H, Chatten J, Newton WAJr., Sachs N, Hamoudi AB, Chiba T, et al. Histopathologic Prognostic Factors in Neuroblastic Tumors: Definition of Subtypes of Ganglioneuroblastoma and an Age-Linked Classification of Neuroblastomas. J Natl Cancer Institute (1984) 73(2):405-16. doi: 10.1093/ jnci/73.2.405

8. Matthay KK, Reynolds CP, Seeger RC, Shimada H, Adkins ES, Haas-Kogan D, et al. Long-Term Results for Children With High-Risk Neuroblastoma Treated on a Randomized Trial of Myeloablative Therapy Followed by 13Cis-Retinoic Acid: A Children's Oncology Group Study. J Clin Oncol (2009) 27(7):1007-13. doi: 10.1200/JCO.2007.13.8925

9. Canete A, Gerrard M, Rubie H, Castel V, Di Cataldo A, Munzer C, et al. Poor Survival for Infants With MYCN-Amplified Metastatic Neuroblastoma Despite Intensified Treatment: The International Society of Paediatric Oncology European Neuroblastoma Experience. J Clin Oncol (2009) 27 (7):1014-9. doi: 10.1200/JCO.2007.14.5839

10. Park JR, Eggert A, Caron H. Neuroblastoma: Biology, Prognosis, and Treatment. Hematology/Oncology Clinics North Am (2010) 24(1):65-86. doi: 10.1016/j.hoc.2009.11.011

11. Maris JM. Recent Advances in Neuroblastoma. N Engl J Med (2010) 362 (23):2202-11. doi: 10.1056/NEJMra0804577

12. Matthay KK, Villablanca JG, Seeger RC, Stram DO, Harris RE, Ramsay NK, et al. Treatment of High-Risk Neuroblastoma With Intensive Chemotherapy, Radiotherapy, Autologous Bone Marrow Transplantation, and 13-CisRetinoic Acid. Children's Cancer Group. N Engl J Med (1999) 341 (16):1165-73. doi: 10.1056/NEJM199910143411601

13. Kushner BH, LaQuaglia MP, Bonilla MA, Lindsley K, Rosenfield N, Yeh S, et al. Highly Effective Induction Therapy for Stage 4 Neuroblastoma in Children Over 1 Year of Age. J Clin Oncol (1994) 12(12):2607-13. doi: 10.1200/JCO.1994.12.12.2607

14. Cheung NK, Kushner BH, LaQuaglia M, Kramer K, Gollamudi S, Heller G, et al. N7: A Novel Multi-Modality Therapy of High Risk Neuroblastoma (NB) in Children Diagnosed Over 1 Year of Age. Med Pediatr Oncol (2001) 36 (1):227-30. doi: 10.1002/1096-911X(20010101)36:1<227::AID-MPO1055>3. $0 . \mathrm{CO} ; 2-\mathrm{U}$

15. Kushner BH, Kramer K, LaQuaglia MP, Modak S, Yataghene K, Cheung NK. Reduction From Seven to Five Cycles of Intensive Induction Chemotherapy in Children With High-Risk Neuroblastoma. J Clin Oncol (2004) 22(24):488892. doi: 10.1200/JCO.2004.02.101

\section{ACKNOWLEDGMENTS}

We thank the anonymous clinicians who participated in this study and the support from CCCG.

16. London WB, Frantz CN, Campbell LA, Seeger RC, Brumback BA, Cohn SL, et al. Phase II Randomized Comparison of Topotecan Plus Cyclophosphamide Versus Topotecan Alone in Children With Recurrent or Refractory Neuroblastoma: A Children's Oncology Group Study. J Clin Oncol (2010) 28(24):3808-15. doi: 10.1200/JCO.2009.27.5016

17. Park JR, Scott JR, Stewart CF, London WB, Naranjo A, Santana VM, et al. Pilot Induction Regimen Incorporating Pharmacokinetically Guided Topotecan for Treatment of Newly Diagnosed High-Risk Neuroblastoma: A Children's Oncology Group Study. J Clin Oncol (2011) 29(33):4351-7. doi: 10.1200/JCO.2010.34.3293

18. Monclair T, Brodeur GM, Ambros PF, Brisse HJ, Cecchetto G, Holmes K, et al. The International Neuroblastoma Risk Group (INRG) Staging System: An INRG Task Force Report. J Clin Oncol (2009) 27(2):298-303. doi: 10.1200/ JCO.2008.16.6876

19. Strother DR, London WB, Schmidt ML, Brodeur GM, Shimada H, Thorner P, et al. Outcome After Surgery Alone or With Restricted Use of Chemotherapy for Patients With Low-Risk Neuroblastoma: Results of Children's Oncology Group Study P9641. J Clin Oncol (2012) 30(15):1842-8. doi: 10.1200/ JCO.2011.37.9990

20. Baker DL, Schmidt ML, Cohn SL, Maris JM, London WB, Buxton A, et al. Outcome After Reduced Chemotherapy for Intermediate-Risk Neuroblastoma. N Engl J Med (2010) 363(14):1313-23. doi: 10.1056/ NEJMoa1001527

21. Brodeur GM, Seeger RC, Barrett A, Berthold F, Castleberry RP, D'Angio G, et al. International Criteria for Diagnosis, Staging, and Response to Treatment in Patients With Neuroblastoma. J Clin Oncol (1988) 6(12):1874-81. doi: 10.1200/JCO.1988.6.12.1874

22. Dueck AC, Mendoza TR, Mitchell SA, Reeve BB, Castro KM, Rogak LJ, et al. Validity and Reliability of the US National Cancer Institute's Patient-Reported Outcomes Version of the Common Terminology Criteria for Adverse Events (PRO-CTCAE). JAMA Oncol (2015) 1(8):1051-9. doi: 10.1001/ jamaoncol.2015.2639

23. Boeva $\mathrm{V}$, Louis-Brennetot $\mathrm{C}$, Peltier $\mathrm{A}$, Durand $\mathrm{S}$, Pierre-Eugène $\mathrm{C}$, Raynal $\mathrm{V}$, et al. Heterogeneity of Neuroblastoma Cell Identity Defined by Transcriptional Circuitries. Nat Genet (2017) 49(9):1408-13. doi: 10.1038/ ng.3921

24. Katzenstein HM, Cohn SL, Shore RM, Bardo DM, Haut PR, Olszewski M, et al. Scintigraphic Response by 123I-Metaiodobenzylguanidine Scan Correlates With Event-Free Survival in High-Risk Neuroblastoma. J Clin Oncol (2004) 22(19):3909-15. doi: 10.1200/JCO.2004.07.144

25. Matthay KK, Edeline V, Lumbroso J, Tanguy ML, Asselain B, Zucker JM, et al. Correlation of Early Metastatic Response by 123I-Metaiodobenzylguanidine Scintigraphy With Overall Response and Event-Free Survival in Stage IV Neuroblastoma. J Clin Oncol (2003) 21(13):2486-91. doi: 10.1200/ JCO.2003.09.122

26. Cheung NV, Heller G. Chemotherapy Dose Intensity Correlates Strongly With Response, Median Survival, and Median Progression-Free Survival in Metastatic Neuroblastoma. J Clin Oncol (1991) 9(6):1050-8. doi: 10.1200/ JCO.1991.9.6.1050

27. van As JW, van den Berg H, van Dalen EC. Different Infusion Durations for Preventing Platinum-Induced Hearing Loss in Children With Cancer. Cochrane Database Systematic Rev (2018) 7(7):Cd010885. doi: 10.1002/ 14651858.CD010885.pub4

28. Brock PR, Maibach R, Childs M, Rajput K, Roebuck D, Sullivan MJ, et al. Sodium Thiosulfate for Protection From Cisplatin-Induced Hearing Loss. N Engl J Med (2018) 378(25):2376-85. doi: 10.1056/NEJMoa1801109

29. Kushner BH, Kramer K, Modak S, Qin LX, Yataghena K, Jhanwar SC, et al. Reduced Risk of Secondary Leukemia With Fewer Cycles of Dose-Intensive Induction Chemotherapy in Patients With Neuroblastoma. Pediatr Blood Cancer (2009) 53(1):17-22. doi: 10.1002/pbc.21931

30. Ladenstein R, Philip T, Lasset C, Hartmann O, Garaventa A, Pinkerton R, et al. Multivariate Analysis of Risk Factors in Stage 4 Neuroblastoma Patients Over the 
Age of One Year Treated With Megatherapy and Stem-Cell Transplantation: A Report From the European Bone Marrow Transplantation Solid Tumor Registry. J Clin Oncol (1998) 16(3):953-65. doi: 10.1200/JCO.1998.16.3.953

31. Harreld JH, Bratton EM, Federico SM, Li X, Grover W, Li Y, et al. Orbital Metastasis Is Associated With Decreased Survival in Stage M Neuroblastoma. Pediatr Blood Cancer (2016) 63(4):627-33. doi: 10.1002/pbc.25847

32. Sokol E, Desai AV, Applebaum MA, Valteau-Couanet D, Park JR, Pearson ADJ, et al. Age, Diagnostic Category, Tumor Grade, and Mitosis-Karyorrhexis Index Are Independently Prognostic in Neuroblastoma: An INRG Project. J Clin Oncol (2020) 38(17):1906-18. doi: 10.1200/JCO.19.03285

33. Park JR, Bagatell R, Cohn SL, Pearson AD, Villablanca JG, Berthold F, et al. Revisions to the International Neuroblastoma Response Criteria: A Consensus Statement From the National Cancer Institute Clinical Trials Planning Meeting. J Clin Oncol (2017) 35(22):2580-7. doi: 10.1200/ JCO.2016.72.0177

34. Genolla J, Rodriguez T, Minguez P, Lopez-Almaraz R, Llorens V, Echebarria A. Dosimetry-Based High-Activity Therapy With (131)IMetaiodobenzylguanidine ((131)I-mIBG) and Topotecan for the Treatment of High-Risk Refractory Neuroblastoma. Eur J Nucl Med Mol Imaging (2019) 46(7):1567-75. doi: 10.1007/s00259-019-04291-x

35. Matthay KK, Tan JC, Villablanca JG, Yanik GA, Veatch J, Franc B, et al. Phase I Dose Escalation of Iodine-131-Metaiodobenzylguanidine With Myeloablative Chemotherapy and Autologous Stem-Cell Transplantation in Refractory Neuroblastoma: A New Approaches to Neuroblastoma Therapy Consortium Study. J Clin Oncol (2006) 24(3):500-6. doi: 10.1200/JCO.2005.03.6400

36. Ladenstein R, Pötschger U, Valteau-Couanet D, Luksch R, Castel V, Yaniv I, et al. Interleukin 2 With Anti-GD2 Antibody Ch14.18/CHO (Dinutuximab Beta) in Patients With High-Risk Neuroblastoma (HR-NBL1/SIOPEN): A Multicentre, Randomised, Phase 3 Trial. Lancet Oncol (2018) 19(12):1617-29. doi: 10.1016/S1470-2045(18)30578-3

37. Yu AL, Gilman AL, Ozkaynak MF, Naranjo A, Diccianni MB, Gan J, et al. Long-Term Follow-Up of a Phase III Study of Ch14.18 (Dinutuximab) + Cytokine Immunotherapy in Children With High-Risk Neuroblastoma: COG Study Anbl0032. Clin Cancer Res (2021) 27(8):2179-89. doi: 10.1158/10780432.CCR-20-3909
38. Matthay KK, Maris JM, Schleiermacher G, Nakagawara A, Mackall CL, Diller L, et al. Neuroblastoma. Nat Rev Dis Primers (2016) 2:16078. doi: 10.1038/nrdp.2016.78

39. Pastor ER, Mousa SA. Current Management of Neuroblastoma and Future Direction. Crit Rev Oncology/Hematology (2019) 138:38-43. doi: 10.1016/ j.critrevonc.2019.03.013

40. Kollareddy M, Sherrard A, Park JH, Szemes M, Gallacher K, Melegh Z, et al. The Small Molecule Inhibitor YK-4-279 Disrupts Mitotic Progression of Neuroblastoma Cells, Overcomes Drug Resistance and Synergizes With Inhibitors of Mitosis. Cancer Lett (2017) 403:74-85. doi: 10.1016/ j.canlet.2017.05.027

41. Heczey A, Louis CU, Savoldo B, Dakhova O, Durett A, Grilley B, et al. CAR T Cells Administered in Combination With Lymphodepletion and PD-1 Inhibition to Patients With Neuroblastoma. Mol Ther J Am Soc Gene Ther (2017) 25(9):2214-24. doi: 10.1016/j.ymthe.2017.05.012

42. Straathof K, Flutter B, Wallace R, Jain N, Loka T, Depani S, et al. Antitumor Activity Without on-Target Off-Tumor Toxicity of GD2-Chimeric Antigen Receptor T Cells in Patients With Neuroblastoma. Sci Trans Med (2020) 12 (571). doi: 10.1126/scitranslmed.abd6169

Conflict of Interest: The authors declare that the research was conducted in the absence of any commercial or financial relationships that could be construed as a potential conflict of interest.

Publisher's Note: All claims expressed in this article are solely those of the authors and do not necessarily represent those of their affiliated organizations, or those of the publisher, the editors and the reviewers. Any product that may be evaluated in this article, or claim that may be made by its manufacturer, is not guaranteed or endorsed by the publisher.

Copyright (c) 2021 Zhang, Kaweme, Duan, Dong and Yuan. This is an open-access article distributed under the terms of the Creative Commons Attribution License (CC BY). The use, distribution or reproduction in other forums is permitted, provided the original author(s) and the copyright owner(s) are credited and that the original publication in this journal is cited, in accordance with accepted academic practice. No use, distribution or reproduction is permitted which does not comply with these terms. 